\title{
On the Benefits of Backup Resource Sharing in Transparent and Opaque Networks
}

\author{
Dimitri Staessens*, Didier Colle*, Mario Pickavet* and, Piet Demeester* \\ * Ghent University - IBBT, Deptartment of Information Technology (INTEC), \\ Gaston Crommenlaan 8 bus 201, 9050 Ghent, Belgium. \\ Email: \{dimitri.staessens, didier.colle, mario.pickavet, piet.demeester\}@intec.ugent.be
}

\begin{abstract}
Transparent networks promise a cost efficient solution for future core and metro networks, due to the high costefficiency for switching trunk traffic. Network availability is an important performance parameter for network operators, who are incorporating protection and restoration mechanisms in the network to achieve competitive advantages. This paper will focus on the reduction in Capital Expenditures (CapEx) expected from implementing protection in transparent networks. We dimension a nationwide transparent network topology for restoration and path protection mechanisms using transparent and opaque architectures. We investigate the gain through protection sharing in relation to the number of links in a meshed network and the offered load on a population of 1000 generated 2-connected planar topologies with 14 nodes. We show that the gain in a transparent network is heavily dependent on the offered load, with almost no relative gain for low load (no required parallel line systems). We also show that, for opaque networks, this relative gain by protection sharing is independent of the traffic load and shows a small dependency on the number of links in the network. The node CapEx reduction for high load is comparable to the CapEx reduction in opaque OTN systems. This is rather surprising as in OTN systems the number of transceivers and linecards and the size of the OTN switching matrix all decrease, while in transparent networks only the degree of the ROADM (number and size of WSSs in the node) decreases while the number of transponders remains the same.
\end{abstract}

\section{INTRODUCTION}

Recent advances made the availability of ultra long haul WDM transmission systems possible at extremely competitive prices. This has opened up new perspectives in the design of cost-effective optical transport networks [1]. Introduction of transparency in the network allows for a reduction in expensive optical-to-electrical-to-optical (OEO) regenerators and effectively reduces the total network cost [2]. According to the utilization of OEO devices, three types of networks are identified: opaque, transparent, and translucent networks. An opaque network is characterized by OEO regenerations at every node. In a transparent network the signal bypasses the OEO devices during its transmission. Translucent networks are situated somewhere inbetween, where some paths require intermediate $\mathrm{OEO}$ regeneration.

One of the key issues in transparent networks is due to the increased length the signal travels without regeneration. Every amplifier adds some noise to the signal, meaning that the signal will have to be regenerated at some point. In addition to this, longer lightpaths are sensitive to various

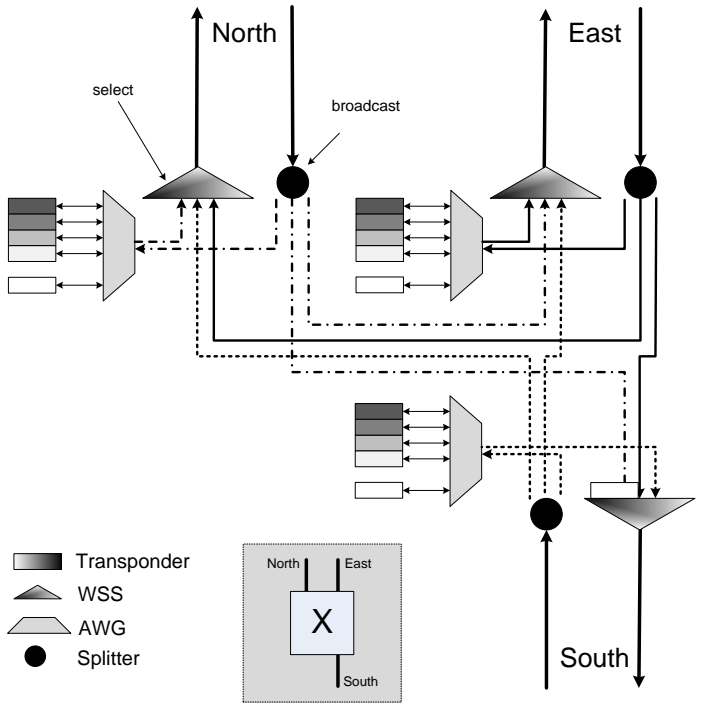

Fig. 1. Reconfigurable Optical Add-Drop Multiplexer Architecture

nonlinear optical impairments, especially when considering high data rates $(>10 \mathrm{~Gb} / \mathrm{s})$. The maximum transparent length (MTL) of a system puts a limit on the size of a completely transparent network. One way of dealing with the impairments in transparent networks is to introduce Islands of Transparency [3]. This is a part of the network where all possible transparent lightpaths are feasible end-to-end. Connections exiting a transparent island are regenerated. A hybrid of the two previous types is called a translucent network where both opaque and transparent functionalities co-exist in a node. In previous work [4] we have shown that sharing backup resources has far less CapEx benefits in transparent networks compared to traditional opaque networks. In this work, we improve and extend that study considerably through simulation on random generated topologies and incorporation of the wavelength continuity constraint, which was not considered in the previous study.

\section{Node Architectures And Cost Model}

The considered transparent node is based on the well-known broadcast-and-select ROADM architecture. Fig. 1 shows a 3-degree ROADM, meaning it has 3 input/output fibers (see simplified structure in the bottom inset) and 3 add/drop terminals. The incoming traffic is split to the other directions 


\begin{tabular}{ll}
\hline Equipment & Cost \\
\hline \hline WDM layer & \\
\hline Transponder 10G grey & 0.1 \\
Transponder 10G 2000km & 1.2 \\
$N$ degree ROADM $(N \leq 8)$ & $N * 9.2$ \\
$N$ degree ROADM $(9 \leq N \leq 19)$ & $N * 11.8$ \\
\hline OTN Layer & \\
\hline Transceiver grey 10G & 0.1 \\
Transceiver 10G 2000km & 1.1 \\
linecard 10x10G & 16 \\
node 8 slot & 7 \\
node 16 slot & 14.3 \\
node 32 slot & 28.6 \\
node 64 slot & 67 \\
node 128 slot & 154
\end{tabular}

TABLE I

COST MODEL

and the add/drop terminal(s). Each transponder is connected via a wavelength multiplexer/de-multiplexer (e.g. AWG) to a fixed direction of the node. If a particular wavelength is not equipped in the terminal for a port, it cannot be used for add/drop at that particular port. The advantage is that there is no need for switching equipment in the add/drop terminals. If we follow the lightpath on the incoming port for an $n$-degree ROADM, it is split to $n$ directions (the $n-1$ outputs and the drop terminal). In the drop terminal it is demultiplexed to the transponders. In the add direction, the transponder output is first aggregated through a multiplexer (AWG) and then selected by a WSS towards the output fiber. The WSS is used to relieve wavelength contention, i.e. if multiple input ports forward traffic on the same wavelength, select the correct one. It could be replaced by a wavelength blocker/filter, which may further reduce costs, however, it seems that commercially it makes little sense as most ROADMs on the market are based on WSS. ROADMs which have degree $N \leq 8$ use $1 \times 9$ WSSs and ROADMs with degree $9 \leq N \leq 19$ use $1 \times 20$ WSSs.

The considered opaque solution is a basic OTN crossconnect, where we consider the costs of the basic node, interface cards and transceivers. For the transparent solution, the cost of the terminals, ROADM node, input amplifiers and transponders are included, as are the tributary interfaces. The cost of the transmission links is not considered in this study, because they will be the same in both solutions. The used cost model is based on the models from [5] [6] [7] and is being updated in the STRONGEST [8] project. The cost values used in this text are given in Table I.

\section{Considered Recovery Methods}

We consider the following protection schemes:

- Unprotected. All traffic is routed over the physical shortest paths, calculated using Dijkstra's algorithm, using $10 \mathrm{G}$ wavelengths.

- Link / node restored. This serves as a benchmark dimensioning for a shared mesh restoration scheme. All traffic is routed over physical shortest paths. For each failure scenario (all possible single link failures for link restored and all possible link and node failures for node-restored) we calculate the required network resources required and determine the minimum which are needed to cover all of the failure scenarios.

- Link / node 1:1 protected. All traffic is routed over physical shortest cycles, calculated using the SuurballeTarjan algorithm [9] for link-disjoint. For node-disjoint, we run the same algorithm on a modified directed graph where each node is split in two nodes, one containing the incoming edges, one containing the outgoing edges and a single directed edge is added between them from the node with the incoming edges to the node with the outgoing edges. The working path is the physically shorter half of the cycle.

- Link / node $1+1$ protected. Uses the same paths as the 1:1 protected, only the traffic is duplicated and sent over both working and backup paths, meaning we also protect the transponders.

In all these scenario's, we use a two-step R+WA approach: we first determine the path using the algorithm detailes above, and then assign the appropriate wavelength(s) using first fit.

Because resource sharing optimization is a complex problem demanding considerable computation resources to find an optimum solution, we use the dimensioning for restoration as a compromise for an optimized shared mesh protection scheme. The drawback of this approach is that it is not feasible to implement restoration on the transparent architecture due to directionality: in the architecture in Fig. 1 we cannot reuse a transponder if its outgoing link fails, because it is tied to this one direction. This means we will have an underestimate of the transponder cost of restoration and 1:1 protection in the transparent solution. In the approach we implemented, due to the possibility that the restoration path for a failed working path can use different outgoing links (as opposed to a single fixed one for 1:1 protection) we underestimate the transponder cost for restoration more than we underestimate the transponder cost for 1:1 protection. This means that, when comparing 1:1 protection to shared mesh protection / restoration in the transparent case, we have an overestimation of the benefits of protection sharing. Also, the $1+1$ protection scheme can be implemented on the ROADM architecture because all transponders are dedicated protected.

We evaluate the cost of these different protection schemes on a national backbone reference network (Fig. 2) with 14 nodes and 23 links. Each link has 80 wavelength channels available. The most relevant characteristics are given in Table II and the traffic matrix is given in III. 


\begin{tabular}{|c|c|c|c|c|c|c|c|c|c|c|c|c|c|c|}
\hline Node ID & 1 & 2 & 3 & 4 & 5 & 6 & 7 & 8 & 9 & 10 & 11 & 12 & 13 & 14 \\
\hline 1 & 0.00 & 8.98 & 12.35 & 13.64 & 9.74 & 32.70 & 19.34 & 21.04 & 14.59 & 33.68 & 15.40 & 12.32 & 23.74 & 11.07 \\
\hline 2 & 8.98 & 0.00 & 5.76 & 6.23 & 4.51 & 14.19 & 9.92 & 10.56 & 6.59 & 12.59 & 6.43 & 5.13 & 10.15 & 4.69 \\
\hline 3 & 12.35 & 5.76 & 0.00 & 12.27 & 10.90 & 21.94 & 11.38 & 13.34 & 12.17 & 17.73 & 9.33 & 7.50 & 15.10 & 6.88 \\
\hline 4 & 13.64 & 6.23 & 12.27 & 0.00 & 12.52 & 24.58 & 12.48 & 14.31 & 18.02 & 19.54 & 10.42 & 8.33 & 16.96 & 7.68 \\
\hline 5 & 9.74 & 4.51 & 10.90 & 12.52 & 0.00 & 17.29 & 8.95 & 10.25 & 10.46 & 13.96 & 7.39 & 5.92 & 11.98 & 5.45 \\
\hline 6 & 32.70 & 14.19 & 21.94 & 24.58 & 17.29 & 0.00 & 28.99 & 33.09 & 27.13 & 47.75 & 26.20 & 21.64 & 27.56 & 19.88 \\
\hline 7 & 19.34 & 9.92 & 11.38 & 12.48 & 8.95 & 28.99 & 0.00 & 20.87 & 13.26 & 26.42 & 13.30 & 10.60 & 20.84 & 9.65 \\
\hline 8 & 21.04 & 10.56 & 13.34 & 14.31 & 10.25 & 33.09 & 20.87 & 0.00 & 15.16 & 30.04 & 14.81 & 11.94 & 23.42 & 10.79 \\
\hline 9 & 14.59 & 6.59 & 12.17 & 18.02 & 10.46 & 27.13 & 13.26 & 15.16 & 0.00 & 20.96 & 11.22 & 8.99 & 18.44 & 8.30 \\
\hline 10 & 33.68 & 12.59 & 17.73 & 19.54 & 13.96 & 47.75 & 26.42 & 30.04 & 20.96 & 0.00 & 22.38 & 18.38 & 34.50 & 16.09 \\
\hline 11 & 15.40 & 6.43 & 9.33 & 10.42 & 7.39 & 26.20 & 13.30 & 14.81 & 11.22 & 22.38 & 0.00 & 10.82 & 20.38 & 10.49 \\
\hline 12 & 12.32 & 5.13 & 7.50 & 8.33 & 5.92 & 21.64 & 10.60 & 11.94 & 8.99 & 18.38 & 10.82 & 0.00 & 16.32 & 7.82 \\
\hline 13 & 23.74 & 10.15 & 15.10 & 16.96 & 11.98 & 27.56 & 20.84 & 23.42 & 18.44 & 34.50 & 20.38 & 16.32 & 0.00 & 17.52 \\
\hline 14 & 11.07 & 4.69 & 6.88 & 7.68 & 5.45 & 19.88 & 9.65 & 10.79 & 8.30 & 16.09 & 10.49 & 7.82 & 17.52 & 0.00 \\
\hline
\end{tabular}

TABLE III

DTAG TOPOLOGY : TRAFFIC MATRIX (GB/s)

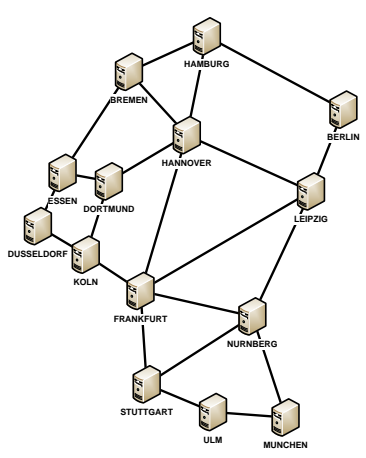

\begin{tabular}{ll}
\hline Node ID & Name \\
\hline 1 & Berlin \\
2 & Bremen \\
3 & Dortmund \\
4 & Düsseldorf \\
5 & Essen \\
6 & Frankfurt/Main \\
7 & Hamburg \\
8 & Hannover \\
9 & Köln \\
10 & Leipzig \\
11 & München \\
12 & Nürnberg \\
13 & Stuttgart \\
14 & Ulm \\
\hline
\end{tabular}

Fig. 2. DTAG reference network

\begin{tabular}{ll}
\hline Parameter & Value \\
\hline Number of Nodes & 14 \\
Number of links & 23 \\
Node degree & $3.29(\min$. 2, Max. 6) \\
Link length $(\mathrm{km})$ & $186 \mathrm{~km}(\min .37$, Max:353 km) \\
Path length $(\mathrm{km})$ & $410 \mathrm{~km}(\min .: 37$, Max.:874) \\
Hop count & $2.35(\min : 1$, Max:5) \\
\hline \multicolumn{3}{c}{ TABLE II } \\
\multicolumn{3}{c}{ DTAG TOPOLOGY CHARACTERISTICS }
\end{tabular}

\section{Dimensioning Results}

\section{A. Link Capacity usage}

Fig. 3 shows the total used link capacity in the network for the different recovery mechanisms. If $w l_{l}^{w}$ is the number of working paths traversing link $l$ and $w l_{l}^{b}$ is the number of backup paths traversing link $l$, the total wavelength consumption for the network with $m$ links is calculated as

$$
\sum_{l=1}^{m} w l_{l}^{w}+w l_{l}^{b}
$$

These values are valid for both the transparent and opaque architectures, as the routing schemes used for both architectures are the same. We clearly see that restoration uses far less wavelengths than protection. Note that for $1+1$ protection the values are the same as for 1:1 protection (the spare capacity in 1:1 protection can of course be used for low priority traffic).

To accomodate all the active lightpaths, the transparent network needs more available channels due to the wavelength continuity constraint. If the wavelength channels on a link are

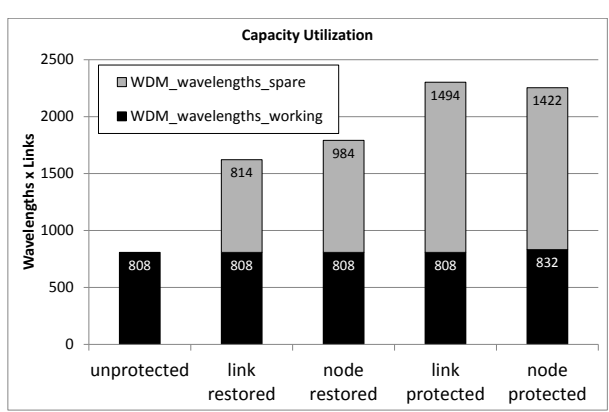

Fig. 3. Link Capacity Utilization for the DTAG Topology

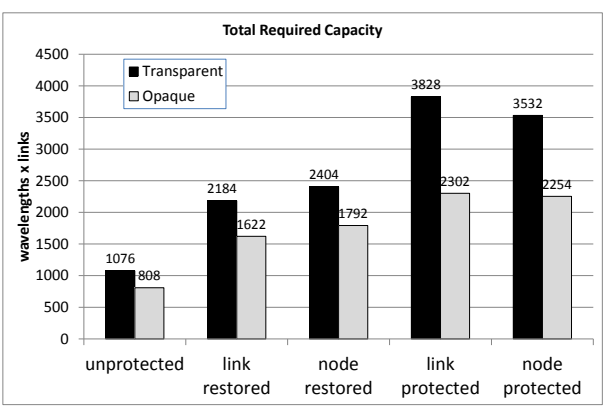

Fig. 4. Link Capacity requirement for the DTAG Topology

numbered starting from 1 in increasing order (for instance, according to the ITU DWDM 50Ghz frequency grid [11]), and the highest used wavelength channel on link $l$ is $w c_{l}$, then the total required network capacity is calculated according to

$$
\sum_{l=1}^{m} w c_{l}
$$

For the opaque solution this amounts to the sum of the working capacity and spare capacity from Fig. 3. We immediately see that, when compared to the opaque solution, the transparent solution requires $33 \%$ more link resources for unprotected traffic, $32 \%$ more for restoration and $66 \%$ more for protection. There is a peculiarity to these results. 


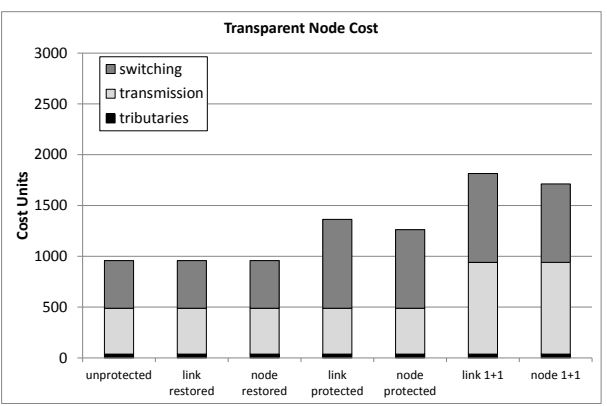

Fig. 5. Transparent Node Cost

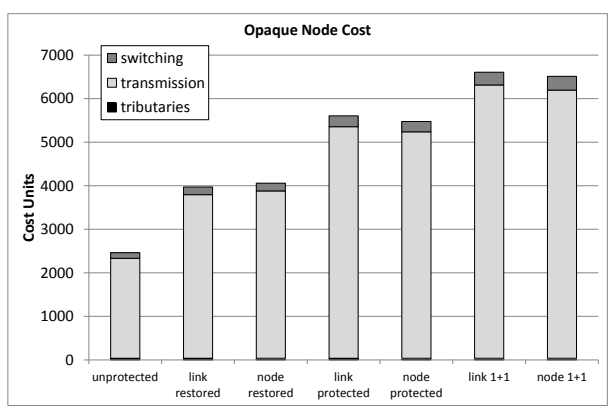

Fig. 6. Opaque Node Cost
The attentive reader will undoubtedly have noticed that, in contradiction to common sense, the link-protected solution consumes more resources than the node-protected solution. This is due to the four nodes in close vicinity of eachother in the DT topology (the link length distribution is not smooth) and the fact that we use a physical length shortest cycle. If we use hopcount instead of physical length in the routing algorithm, this does not occur.

\section{B. Node Capital Expenditures}

Now we turn our attention to the Capital Expenditures (CapEx) of the nodes. The CapEx of the nodes is broken down in three main components:

- Tributaries. The transmission equipment (transponders or transceivers) towards the client host or network).

- Transmission. These are the source and destination transponders (transparent) / transceivers (opaque), any intermediate transponders/transceivers and the OTN linecards (opaque).

- Switching. These are the switching fabric and AWG/terminals in the ROADM or the backplane in the OTN cross-connect.

Figs. 5 and 6 show the results for the transparent and opaque solutions respectively. It is immediately clear that the restoration (i.e. the solution with backup capacity sharing) is cheaper than protection in both solutions. In the transparent network, the cost difference is in the switching. If the capacity (in this case needed for recovery purposes) increases, the capacity of some links my exceed the number of wavelengths (80), so some nodes need a parallel line system in order to accomodate this increase in traffic. For the opaque solution, the main cost is in the transmission equipment because we need 2 transceivers in every intermediate node for each traversing connection. There is also an increase in the switching cost due to larger backplane requirements for protection when compared to restoration. Also, in the opaque solution, the cost of the tributaries is very small compared to the overall node cost.
If we compare the gain by implementing protection sharing, we see that for the opaque solution, the gain is roughly $43 \%$, while for the transparent solution it is roughly $36 \%$. This result seems to be in contradiction with our result from [4], where we found no such advantage for the traffic from Table III and only a little advantage if we doubled the amount of traffic in the traffic matrix. The reason for the discrepancy lies in the fact that we did not take into account the wavelength continuity constraint in our previous work. As was shown in Fig. 3 the continuity constraint leads to almost $50 \%$ increase in traffic for the restoration case and a $75 \%$ increase in traffic for the protection case, effectively increasing the reduction in node CapEx gained by resource sharing in transparent networks. While we expected a small increase, we never expected such a significant one. This find leads us to perform more extensive research in order to find the relation between the traffic and the node cost for transparent and opaque networks. In the next section, we perform a thorough investigation how the node CapEx gain (through the introduction of resource sharing) scales with traffic demand and network meshedness.

\section{RANDOMIZED CONTROL STUDY}

In order to have a more meaningful analysis and evaluate the benefits of resource sharing more throughly, we extend our dimensioning study by using random generated 14-node networks as opposed to a single reference network. We number the nodes 1-14 at random, apply the traffic matrix from Table III to each of these networks and analyze the node CapEx.

We generated 2-node-connected planar graphs by randomly assigning 14 points to a $800 \mathrm{~km}$ by $800 \mathrm{~km}$ grid and computing the Gabriel graph [10] for these 14 points. We discarded all non 2-connected graphs until we had a population of 1000 random graphs. These graphs had a link distribution shown in Fig. 7. It seems that the topology with 23 nodes (like the DTAG topology) is the most likely to occur. 


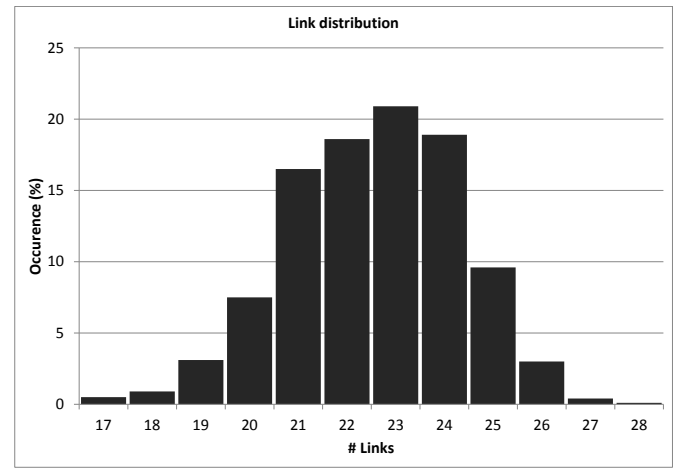

Fig. 7. Link Distribution for the generated topologies

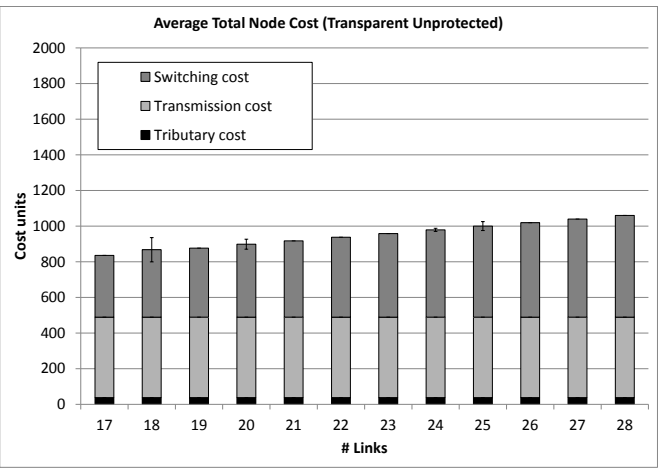

Fig. 8. Average Node Cost, Transparent Unprotected

\section{A. Influence of the Topology}

Fig. 8 shows the average node costs for the generated networks versus the number of links in the generated topologies for unprotected routing. $2 \sigma$ confidence intervals are included (note than there is no variation for most of the unprotected networks). The cost of a transparent network seems to go up with the number of available links. From Fig. 8 we clearly see that this is due to an increase in switching cost, or more specifically, an increase in the degree of the ROADM node due to the increase in physical degree of the topology. We will investigate the effect of traffic increases later. For some networks (the 18,20,24 and 25) there is a slight variation in the cost of the switch due to some distributions requiring parallel line systems.

Figs. 9 and 10 show similar figures for node restoration and protection. We see that the monotonous increase of the cost vs the number of links observed for the unprotected case is not here anymore and shows a more flat distribution. If we look at the cost benefits of protection vs restoration (i.e. the difference between Fig. 10 and Fig. 9 we see that for the 23 link network, in our generated topologies the gain is around $22 \%$. What is very peculiar is that the gain is higher for the medium meshed networks (21-24 link networks are all in the $20-25 \%$ range) than for the higher meshed networks (the gain for the 26 links network is already less

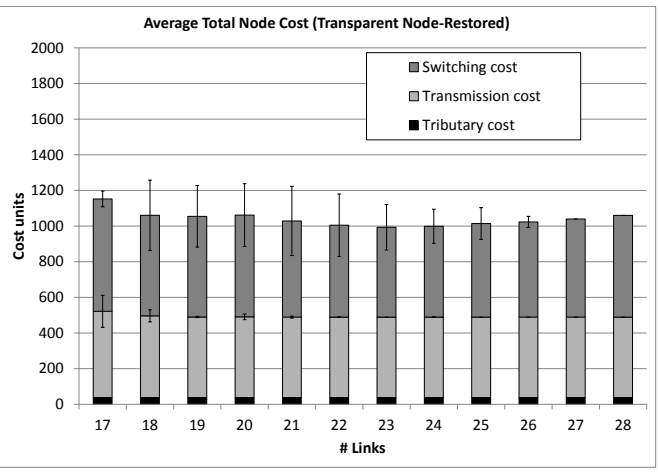

Fig. 9. Average Node Cost, Transparent Node-Restored

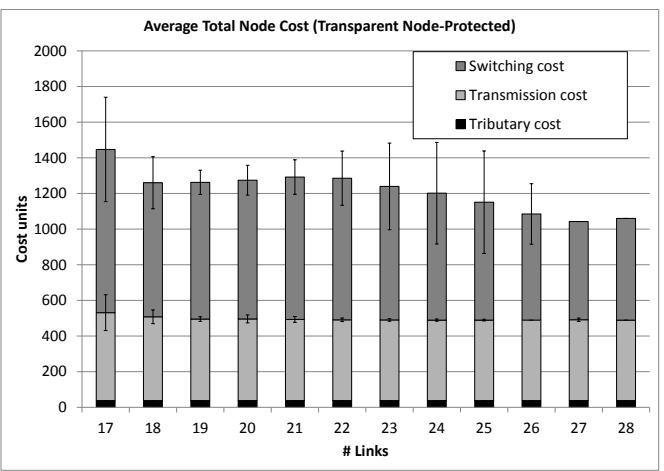

Fig. 10. Average Node Cost, Transparent Node-Protected

than $10 \%$ ). In these figures, we also notice some variation in the transmission cost. This is because the networks are not transparent anymore for the restoration paths (they are for the unprotected shortest paths) and in some networks the longer paths require regeneration, which is implemented by terminating and continuing the traffic at an intermediate node.

We now turn our attention to the opaque architecture. Figs. 11 and 12 show the node costs for the node-restored and node-protected cases. We see that the cost of the network scales down with an increase in the number of links. This is because an increase in meshedness reduces the average hops on each path, which in turn reduces the number of OEO conversions and therefore the transmission cost.

When we compare the two solutions, we again see a node CapEx gain which decreases with the number of links in the network. The gain is $25 \%$ for a 17 link network and $21 \%$ for a 28 link network.

\section{B. Influence of Traffic Scaling}

In order to evaluate the effects of the traffic load, we scaled the traffic from Table III from 50\% to $500 \%$ in $50 \%$ increments. From a multiplier of 3-3.5x onwards, the ROADM degree of some node exceeds 19 , and the OTN 


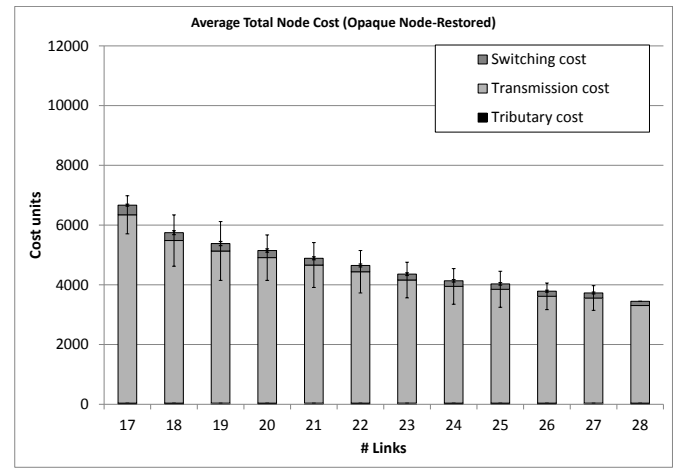

Fig. 11. Average Node Cost, Opaque Node-Restored

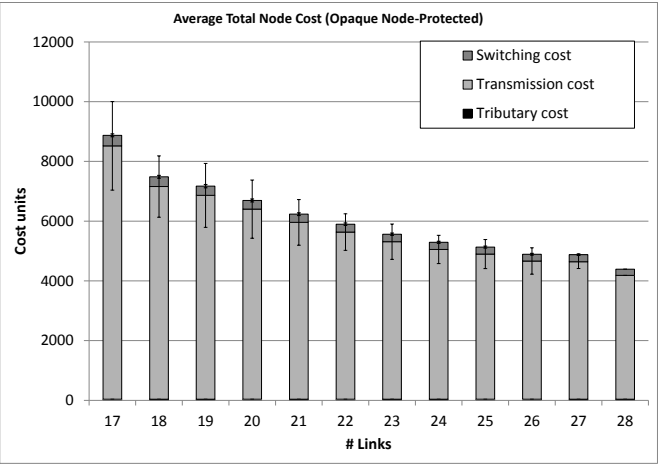

Fig. 12. Average Node Cost, Opaque Node-Protected

backplane reaches its limits at 4.5-5x. We therefore limit our results to a traffic multiplier for $3 \mathrm{x}$ for the transparent case and $4.5 \mathrm{x}$ for the opaque case.

From Fig. 13 and 14 it is clear that the increase in node cost with the number of links we noticed in the previous subsection is only valid for the low traffic cases (multiplier $0.5 \mathrm{x}$ and $1 \mathrm{x}$ ), where there is little increase in ROADM degrees through the necessity for additional parallel line systems.

We summarize the relative CapEx gain for the transparent networks in Fig. 15. We include the networks with 20-25 links and apply the traffic multiplier from $0.5 x$ to $3 x$. We omit the other cases because of some ROADM degrees exceeding 19 as noticed before. What we learn from this figure is that the relative gain through protection sharing in transparent networks is very dependent on the traffic scaling. For low traffic there is almost no resource gain. From the moment the traffic loads exceeds a certain threshold (here it's roughly at the $1 \mathrm{x}$ multiplier) and the average ROADM degree in the network goes up, the relative decrease in traffic load needed to reduce the degree goes down and the probability of this happening goes up significantly. If we have a node with 3 neighbours in the physical topology, reducing it from a 14-degree to a 12-degree ROADM takes less of a relative traffic reduction than to reduce it from a 4-degree to a 3-degree ROADM. The

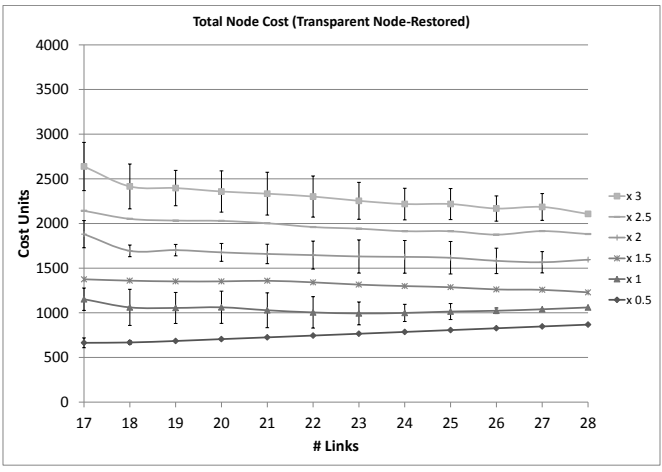

Fig. 13. Total Node Cost for Different Traffic Multipliers, Transparent NodeRestored

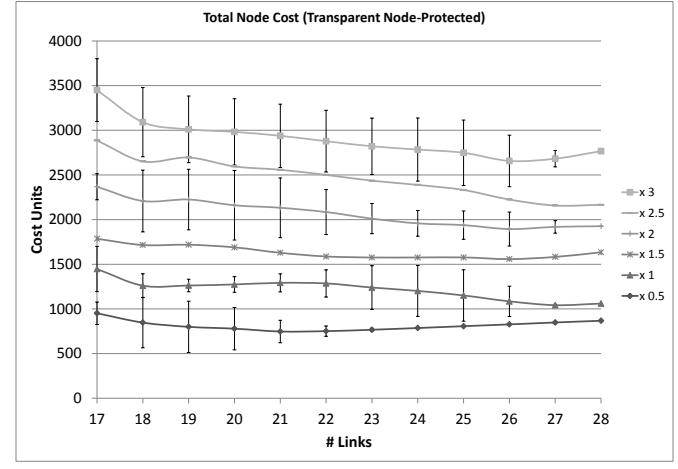

Fig. 14. Total Node Cost for Different Traffic Multipliers, Transparent NodeProtected

wavelength continuity constraint is certainly an important contributor in speeding up this process. After this threshold is reached the CapEx gain of resource sharing is rought $17-22 \%$.

For opaque networks, the overall picture is quite different. As shown in Figs. 16 and 17, the total node cost always goes down with the number of links in the network, no matter the load. Also, it seems to scale with the load, so the higher the load, the more beneficial to install additional links. This means operators will be able to find an optimum between the additional link cost and the decreasing node cost. Notice that transparent networks with low traffic do not have this and have a decrease in node cost together with a decrease in link cost, always driving the optimum towards sparsely meshed networks. This may give transparent network operators additional incentives to prefer higher bandwidths per channel and more wavelengths per fiber instead of installing parallel line systems.

When comparing the overall cost reduction from Figure 15 and 18 we see that the node CapEx reduction for transparent networks in the high load case (17-22\%) is definitely comparable to the CapEx reduction in the opaque architecture $(21-25 \%)$. We find this quite surprising as the load reduction through resource sharing in transparent networks only affects 


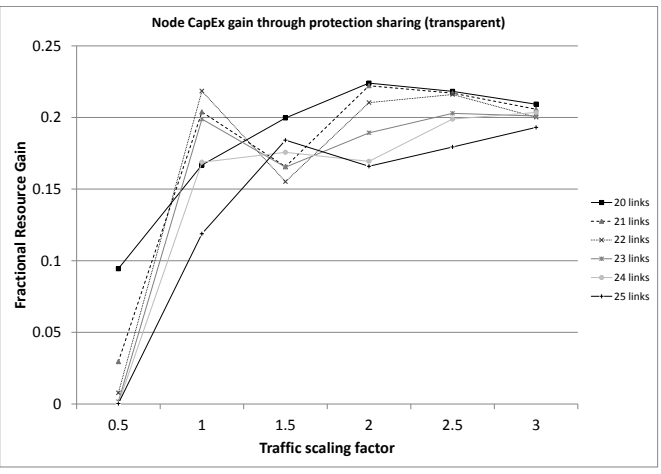

Fig. 15. CapEx gain through Resource Sharing in Transparent Networks

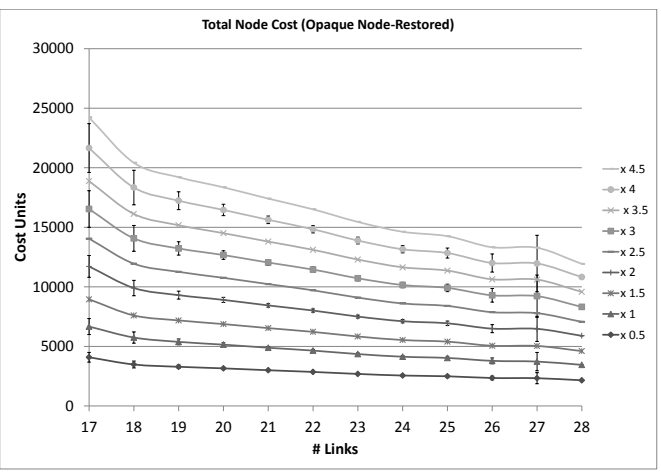

Fig. 16. Total Node Cost for Different Traffic Multipliers, Opaque NodeRestored

the ROADM degree and therefore only the cost of the WSS drives this reduction. In opaque networks the cost reduction is driven through a reduction in the number of required transceivers, linecards and a reduction in the size of the switching fabrix.

\section{CONClusions AND Future Work}

In this work, we performed a thorough investigation into the possible CapEx saving through resource sharing in transparent and opaque transport networks. The number of links in the network have a small impact on this gain, with sparsely meshed networks having greater benefit than densely meshed networks. We find that the load has an important impact in transparent networks, where low load (i.e. few parallel line systems) means that the network does benefit greatly from protection sharing. However, when the average required ROADM degree increases, the CapEx benefits approach the same levels as for traditional opaque networks. Opaque networks do not show a dependency on the load and always have a similar node CapEx gain from protection sharing.

\section{ACKNOWLEDGMENT}

This work was supported by the European Commission within the STRONGEST-project (Scalable, Tunable and Resilient Optical Networks Guaranteeing Extremely-high Speed

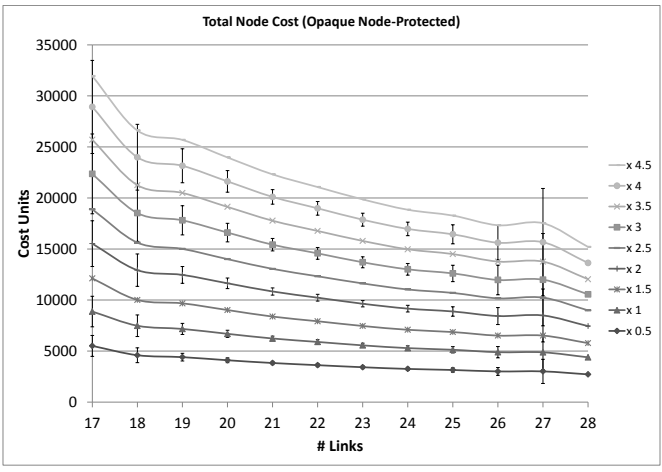

Fig. 17. Total Node Cost for Different Traffic Multipliers, Opaque NodeProtected

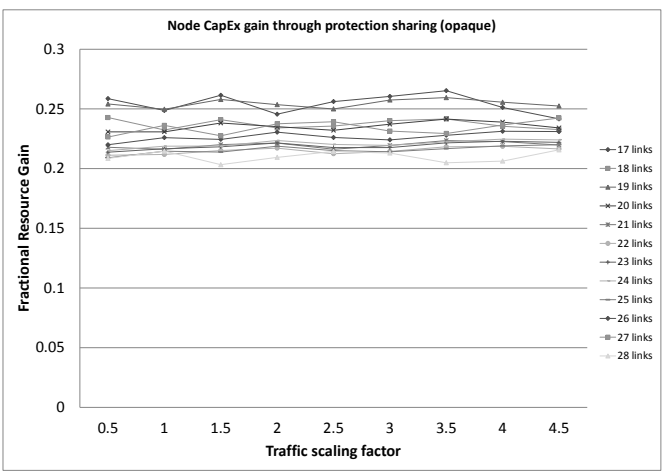

Fig. 18. CapEx gain through Resource Sharing in opaque Networks

Transport), funded through the 7th ICT-Framework Programme.

\section{REFERENCES}

[1] B. Ramamurthy et al., Transparent vs. opaque vs. translucent wavelength-routed optical networks, Proc. OFC'99 Feb. 1999.

[2] A. Morea et al., A critical analysis of the possible cost savings of translucent networks, Proc. DRCN 2005.

[3] R. D. Doverspike et al., Future Transport Network Architectures, IEEE Communications Magazine,Special Issue on Reliable Communication Networks, Aug. 1999.

[4] D. Staessens et al. Cost Efficiency of Protection in Future Transparent Networks, Proc. ICTON2009, July 2009.

[5] M. Gunkel et al., A Cost Model for the WDM Layer, in Proc. Photonics in Switching 2006

[6] R. Huelsermann et al., Cost modeling and evaluation of capital expenditures in optical multilayer networks, OSA J. of Optical Networking, vol. 7, no. 9, 2008

[7] M. De Groote et al., Cost comparison of different Translucent Optical Network Architectures, in Proc. CTTE 2010.

[8] STRONGEST: Scalable, Tunable and Resilient Optical Networks Guaranteeing Extremely-high Speed Transport, http://www.ict-strongest.eu/.

[9] J. W. Suurballe and R. E. Tarjan,A quick method for finding shortest pairs of disjoint paths Networks 14, pp. 325336, 1984.

[10] K. R. Gabriel and R. R. Sokal, A new statistical approach to geographic variation analysis, Systematic Zoology (Society of Systematic Biologists) 18 (3) pp. 259270, 1969

[11] ITU-T recommendation 694.1 Spectral grids for WDM applications: DWDM frequency grid, http://www.itu.int. 